\title{
An Application of Dynamic Programming Principle in Corporate International Optimal Investment and Consumption Choice Problem
}

\author{
Zongyuan Huang and Zhen Wu \\ School of Mathematics, Shandong University, Jinan 250100, China \\ Correspondence should be addressed to Zhen Wu, wuzhensdu@gmail.com
}

Received 5 August 2010; Revised 16 December 2010; Accepted 27 December 2010

Academic Editor: Gradimir V. Milovanović

Copyright (c) 2010 Z. Huang and Z. Wu. This is an open access article distributed under the Creative Commons Attribution License, which permits unrestricted use, distribution, and reproduction in any medium, provided the original work is properly cited.

This paper is concerned with a kind of corporate international optimal portfolio and consumption choice problems, in which the investor can invest her or his wealth either in a domestic bond (bank account) or in an oversea real project with production. The bank pays a lower interest rate for deposit and takes a higher rate for any loan. First, we show that Bellman's dynamic programming principle still holds in our setting; second, in terms of the foregoing principle, we obtain the investor's optimal portfolio proportion for a general maximizing expected utility problem and give the corresponding economic analysis; third, for the special but nontrivial Constant Relative Risk Aversion (CRRA) case, we get the investors optimal investment and consumption solution; last but not least, we give some numerical simulation results to illustrate the influence of volatility parameters on the optimal investment strategy.

\section{Introduction}

International portfolio diversification problem plays an important role in finance and generally depends on several factors such as barriers of international capital markets, uncertainty of operational cash flows, and the effect of exchange risk on corporate international investment. Some more theories and models can be found in Choi [1]. Since Duffie [2] and Merton [3] originally introduced stochastic models of security markets, optimal portfolio and consumption choice problems have been studied by many researchers. Zariphopoulou [4,5] studied investment and consumption models with transactions fees and some trading constraints. Bellalah and $\mathrm{Wu}$ [6] gave a simple model of corporate international investment under incomplete information and taxes. Wang and $\mathrm{Wu}$ [7] obtained a general maximum principle for partially observed risk-sensitive optimal control problems 
and applied it to solve some problems arising from financial market. In 1997, El Karoui et al. [8] used backward stochastic differential equation (BSDE) theory to investigate some options pricing problem with the borrowing rate higher than the deposit rate.

For most investors, the profit is the most important while the risk cannot be ignorable. Since the investors who want to invest in an international corporate will face more liquidity risk, more risk in exchange rate, they usually choose a securer way in their domestic investment, which could offer enough liquidity and stable gains, such as bonds and other fixed-income derivatives. On the other hand, although bonds have lower yields, they have been widely selected as part of a portfolio from the viewpoint of risk management. In this paper, we are concerned with a kind of optimal international corporate portfolio and domestic consumption choice problem. Under our framework, an investor has two choices: one is a bond (bank account) in home country and the other is a real project with production in foreign country. The investor can borrow money from the bank to invest in the real project if he pays higher loan rate than deposit, meanwhile, the investor is also supposed to retain her/his domestic consumption habit. This reflects an implicit assumption according to which all the foreign projects are evaluated in foreign currency and converted by uncertain exchange rate. In order to maximize her/his expected utility of both consumption and her/his terminal wealth in domestic currency numeraire, the problem is then formulated as a kind of optimal control problems. To deal with this kind of problems, an efficient method is to apply Bellman's dynamic programming principle which was originally founded in 1952 (see Bellman [9]). This principle implies that an optimal policy has the property that whatever the initial state and initial decision are, the remaining decisions must constitute an optimal policy with regard to the state resulting from the first decision. Since it was founded, this principle has a broad applications in lots of different fields, such as in bank management and in insurance (see Mukuddem-Petersen and Petersen [10,11]).

The paper is organized as follows. In Section 2, we formulate the maximizing expected utility problem and give some corresponding assumptions. Section 3 is devoted to establish the corresponding Bellmans dynamic programming principle and use it to solve the foregoing problem. In addition, some economic analysis on the optimal solutions is also given in this section. Section 4 concerns a special but nontrivial CRRA case. By a conjecture method arising from the classical linear quadratic (LQ) optimal control problems, the explicit optimal investment proportion and consumption choice are derived. Furthermore, some numerical simulations are used to explicitly illustrate the influence of the volatility parameters in foreign production and exchange rate market on the optimal investment strategy. Finally, we give some concluding remarks.

\section{Model and Formulation of the Problem}

Let $(\Omega, \mathcal{F}, P)$ be a complete probability space endowed with a filtration $\left\{\mathcal{F}_{t} ; 0 \leq t \leq T\right\}$. $\{B(t)\}_{0 \leq t \leq T}$ and $\{\bar{B}(t)\}_{0 \leq t \leq T}$ are two one-dimensional Brownian motions defined on this space, which represents the external sources of uncertainty in the markets with the correlation coefficient $\rho$.

Suppose the investor can put his money in the bank account to get nonrisk reward, and the price of the bank account satisfies the equation

$$
d P_{0}(t)= \begin{cases}r(t) P_{0}(t) d t, & \text { if } P_{0}(t) \geq 0 \\ r^{\prime}(t) P_{0}(t) d t, & \text { if } P_{0}(t)<0\end{cases}
$$


where $r(t)$ is the interest rate and $r^{\prime}(t)$ is the borrowing rate for any loan. Moreover, according to the real market situation, the borrowing rate $r^{\prime}(t)$ should be a bit larger, that is, $r^{\prime}(t) \geq$ $r(t)$, a.s..

On the other hand, the investor can choose a foreign real project with some production to get a higher return but with risk. The cash flow from this project in foreign currency numeraire is

$$
\tilde{R}_{c f}(t)=(1-\tau)[S(t)-P(t)] Q(t),
$$

with

$$
Q(t)=[S(t)]^{\beta}
$$

Here $P(t)$ and $S(t)$ are input price and output price of the production, $Q(t)$ is the quantity of the output. $\beta$ is a negative constant in general, and $\tau$ is the tax rate in the foreign country market.

Suppose that the input price and output price are described as

$$
\begin{aligned}
& d P(t)=\alpha_{P}(t) P(t) d t+\sigma(t) P(t) d B(t), \\
& d S(t)=\alpha_{S}(t) S(t) d t+\sigma(t) S(t) d B(t),
\end{aligned}
$$

where $P(t), S(t)$ are with the initial values $P(0), S(0) . e(t)$ is the exchange rate from foreign currency to domestic currency, and

$$
d e(t)=\alpha_{e}(t) e(t) d t+\sigma_{e}(t) e(t) d \bar{B}(t) .
$$

In the actual long time duration market, there is a mean reversion on the exchange rate. In our solving frame, we use a geometric Brownian motion to describe the exchange rate in a specified short time duration market to get an explicit optimal solution.

Due to the above expressions, it is easy to derive

$$
\begin{aligned}
& P(t)=P(0) e^{\int_{o}^{t} \sigma(s) d B(s)} e^{\int_{0}^{t}\left[\alpha_{P}(s)-(1 / 2) \sigma^{2}(s)\right] d s}, \\
& S(t)=S(0) e^{\int_{o}^{t} \sigma(s) d B(s)} e^{\int_{0}^{t}\left[\alpha_{S}(s)-(1 / 2) \sigma^{2}(s)\right] d s} .
\end{aligned}
$$

Then

$$
\tilde{R}_{c f}(t)=(1-\tau) S^{\beta}(t)[S(t)-P(t)]=(1-\tau)[S(0)]^{\beta} e^{(1+\beta) \int_{0}^{t} \sigma(s) d B(s)} F(t),
$$

where

$$
F(t)=e^{\beta \int_{0}^{t}\left[\alpha_{S}(s)-(1 / 2) \sigma^{2}(s)\right] d s}\left[S(0) e^{\int_{0}^{t}\left[\alpha_{S}(s)-(1 / 2) \sigma^{2}(s)\right] d s}-P(0) e^{\int_{0}^{t}\left[\alpha_{P}(s)-(1 / 2) \sigma^{2}(s) d s\right.}\right] .
$$


Applying Itô's formula to $\widetilde{R}_{c f}(t)$, we can get

$$
d \widetilde{R}_{c f}(t)=\widetilde{R}_{c f}(t)(1+\beta) \sigma(t) d B(t)+\widetilde{R}_{c f}(t) f(t) d t
$$

where $f(t)=(1 / 2)(1+\beta)^{2} \sigma^{2}(t)+F^{\prime}(t) / F(t), F(t) \neq 0$. That is, the input price is not equal to the output price.

We translate the cash flow to domestic currency by exchange rate and let $R_{c f}(t)=$ $e(t) \widetilde{R}_{c f}(t)$; then

$$
d R_{c f}(t)=g(t) R_{c f}(t) d t+(1+\beta) \sigma(t) R_{c f}(t) d B(t)+\sigma_{e}(t) R_{c f}(t) d \bar{B}(t),
$$

where $g(t)=f(t)+\alpha_{e}(t)+(1+\beta) \rho \sigma_{e}(t) \sigma(t)$.

Let $W(t)$ denote the total wealth at time $t$ and $\pi(t)$ the amount investing in foreign market, then $W(t)-\pi(t)$ is the amount investing in non-risky bond. If $C(t)$ stands for the consumption of the investor, then the change of the wealth would include three parts, the change of risky part $d \pi(t)$ and the risk-free part $d(W(t)-\pi(t))$, and also the consumption $d C(t)=c(t) d t, c(t)$ is the consumption rate. Finally it follows the equation

$$
\begin{aligned}
d W(t)= & {\left[r(t) W(t)-c(t)+(g(t)-r(t)) \pi(t)-\left(r^{\prime}(t)-r(t)\right)(W(t)-\pi(t))^{-}\right] d t } \\
& +\sigma(t) \pi(t)(1+\beta) d B_{t}+\pi(t) \sigma_{e}(t) d \bar{B}(t) .
\end{aligned}
$$

Note that $(W(t)-\pi(t))^{-}$denotes the negative part of $W(t)-\pi(t)$, whose value is $\pi(t)-W(t)$ when $W(t)<\pi(t)$ and 0 otherwise. It is not hard to see that only when the investor takes loan from bank, this part is taken into account.

Let us use the functions $l$ and $h$ to denote the investor's preference with respect to consumption and terminal wealth and the integral of the cumulated utility $l$ from the consumption. Suppose the investor wants to maximize the expected utility

$$
J(c, \pi, W)=E\left[\int_{0}^{T} l(c(t), t) d t+h(W(T), T)\right],
$$

with the following assumption conditions

(A1) $l, h$ are strictly increasing functions;

(A2) $l, h$ are concave and differentiable w.r.t. $c$ and $W$.

Note that these assumptions are common properties of utility function which can be seen in Karatzas [12]. The above formulates one kind of optimal control problems. Then the rest is devoted to solving it. 


\section{Dynamic Programming Principle and the Optimal Solutions}

In this section, we will show that the celebrated dynamic programming principle still holds for the optimization problem (2.12) and use it to give the optimal investment and consumption choice of the general case, then give the corresponding economic analysis.

The basic idea of the dynamic programming principle is to consider a family of optimal control problems and establish the relationships among them via the HamiltonJacobi-Bellman (HJB) equation. If the HJB equation is solvable, then we can obtain the desired optimal control. This is the so-called verification technique. As we know, dynamic programming method is now widely used to deal with some control problems, either deterministic or stochastic. (More results can be found for example, in Bellman [13], Peng [14], El Karoui and Quenez [15], etc.).

For any $0 \leq s \leq T$, define the value function

$$
V(W, s)=\sup _{(c, \pi)} J(c, \pi, W, s)=\sup _{(c, \pi)} E\left[\int_{s}^{T} l(c(t), t) d t+h(W(T), T)\right]
$$

and denote that $\mathcal{F}_{t}^{s}=\sigma\{(B(r)-B(s), \bar{B}(r)-\bar{B}(s)): s \leq r \leq t\}$.

We state the following dynamic programming principle for our optimal control problem which can be obtained by Lemma 3.2 of Chapter 4 in Yong and Zhou [16].

Proposition 3.1. Let (A1) and (A2) hold. Then for any $(W, s) \in \mathbb{R} \times[0, T)$,

$$
V(W, s)=\sup _{(c, \pi)} E\left[\int_{s}^{\widehat{s}} l(c(t), t) d t+V\left(W_{\widehat{s}}, \widehat{s}\right)\right], \quad \forall 0 \leq s \leq \widehat{s} \leq T .
$$

Now we give the corresponding HJB equation.

Proposition 3.2. Under the assumptions (A1) and (A2), if $V(W, s) \in C^{2,1}(\mathbb{R} \times[0, T])$, then it is the solution of the following partial differential equation (HJB equation):

$$
\begin{gathered}
\frac{\partial V}{\partial s}+\frac{\partial V}{\partial W} r(s) W+\sup _{c}\left\{l(c(s), s)-\frac{\partial V}{\partial W} c(s)\right\} \\
+\sup _{\pi}\left\{\frac{\partial V}{\partial W} \pi(s)(g(s)-r(s))-\frac{\partial V}{\partial W}\left(r^{\prime}(s)-r(s)\right)(W(s)-\pi(s))^{-}\right. \\
\left.+\frac{1}{2} \frac{\partial^{2} V}{\partial W^{2}}\left[(1+\beta)^{2} \sigma^{2}(s) \pi^{2}(s)+\pi^{2}(s) \sigma_{e}^{2}(s)+2(1+\beta) \sigma(s) \sigma_{e}(s) \rho \pi^{2}(s)\right]\right\}=0, \\
V(W, T)=h(W, T) .
\end{gathered}
$$


Proof. For fixed $(W, t) \in \mathbb{R} \times[0, T)$, applying Itô's formula to $V(W, s)$ and then sending $t$ to $s$, we can take the same steps as that of Proposition 3.5 of Chapter 4 in Yong and Zhou [16] to get the desired result.

The above proposition implies that if we could obtain the solution $V(W, s)$ to the HJB equation, then we could construct an optimal control for the problem (2.11)-(2.12). This is called the verification technique, which is the chief method to solve this kind of optimization problems using dynamic programming principle.

According to the assumption of Proposition 3.2, it is natural to suppose that $\partial V / \partial W \geq 0$ and $\partial^{2} V / \partial W^{2}<0$. This property is satisfied in many typical utility function cases such as CRRA case in the next section. This also means that the value function is nondecreasing and concave.

Suppose that the optimal consumption rate choice $c^{*}$ together with optimal proportion $\pi^{*}$ is the optimal solution to our problem, then $c^{*}(s)=l_{c}^{-1}(\partial V / \partial W, s)$, where $l_{c}^{-1}$ denotes the inverse function of the consumption marginal utility.

Let us define

$$
\begin{aligned}
k(\pi):= & \frac{\partial V}{\partial W}(g(s)-r(s)) \pi(s)+\frac{1}{2} \frac{\partial^{2} V}{\partial W^{2}}\left[(1+\beta)^{2} \sigma^{2}(s)+\sigma_{e}^{2}(s)+2 \rho(1+\beta) \sigma(s) \sigma_{e}(s)\right] \pi^{2}(s) \\
& -\frac{\partial V}{\partial W}\left(r^{\prime}(s)-r(s)\right)(W(s)-\pi(s))^{-}, \\
k_{1}(\pi):= & \frac{\partial V}{\partial W}(g(s)-r(s)) \pi(s)+\frac{1}{2} \frac{\partial^{2} V}{\partial W^{2}}\left[(1+\beta)^{2} \sigma^{2}(s)+\sigma_{e}^{2}(s)+2 \rho(1+\beta) \sigma(s) \sigma_{e}(s)\right] \pi^{2}(s), \\
k_{2}(\pi):= & \frac{\partial V}{\partial W}\left(g(s)-r^{\prime}(s)\right) \pi(s)+\frac{1}{2} \frac{\partial^{2} V}{\partial W^{2}}\left[(1+\beta)^{2} \sigma^{2}(s)+\sigma_{e}^{2}(s)+2 \rho(1+\beta) \sigma(s) \sigma_{e}(s)\right] \pi^{2}(s) \\
& +\frac{\partial V}{\partial W}\left(r^{\prime}(s)-r(s)\right) W(s),
\end{aligned}
$$

then

$$
k(\pi)= \begin{cases}k_{1}(\pi), & W \geq \pi \\ k_{2}(\pi), & W<\pi\end{cases}
$$

We have

$$
\sup _{\pi} k_{1}(\pi)=-\frac{1}{2} \frac{(\partial V / \partial W)^{2}(g(s)-r(s))^{2}}{\left(\partial^{2} V / \partial W^{2}\right)\left[(1+\beta)^{2} \sigma^{2}(s)+\sigma_{e}^{2}(s)+2 \rho(1+\beta) \sigma(s) \sigma_{e}(s)\right]}
$$


with maximal point

$$
\begin{aligned}
\pi_{1}^{*}(s)= & -\frac{(\partial V / \partial W)(g(s)-r(s))}{\left(\partial^{2} V / \partial W^{2}\right)\left[(1+\beta)^{2} \sigma^{2}(s)+\sigma_{e}^{2}(s)+2 \rho(1+\beta) \sigma(s) \sigma_{e}(s)\right]} \\
\sup _{\pi} k_{2}(\pi)= & -\frac{1}{2} \frac{(\partial V / \partial W)^{2}\left(g(s)-r^{\prime}(s)\right)^{2}}{\left(\partial^{2} V / \partial W^{2}\right)\left[(1+\beta)^{2} \sigma^{2}(s)+\sigma_{e}^{2}(s)+2 \rho(1+\beta) \sigma(s) \sigma_{e}(s)\right]} \\
& +\frac{\partial V}{\partial W}\left(r^{\prime}(s)-r(s)\right) W(s)
\end{aligned}
$$

with maximal point

$$
\pi_{2}^{*}(s)=-\frac{(\partial V / \partial W)\left(g(s)-r^{\prime}(s)\right)}{\left(\partial^{2} V / \partial W^{2}\right)\left[(1+\beta)^{2} \sigma^{2}(s)+\sigma_{e}^{2}(s)+2 \rho(1+\beta) \sigma(s) \sigma_{e}(s)\right]} .
$$

Noting the assumption $\partial V / \partial W \geq 0, \partial^{2} V / \partial W^{2}<0$, we then get that $\pi_{1}^{*}(s) \geq \pi_{2}^{*}(s)$.

Now, let us discuss the optimal portfolio choice based on the relationship between $\pi^{*}$ and $W$.

Case $1\left(W(s) \leq \pi_{2}^{*}(s)\right)$. From Figure 1 , we can conclude that $W \leq \pi_{2}^{*} \leq \pi_{1}^{*}$, then

$$
\pi^{*}(s)=\pi_{2}^{*}(s)
$$

The corresponding HJB equation is

$$
\begin{gathered}
\frac{\partial V}{\partial s}+\frac{\partial V}{\partial W} r^{\prime}(s) W+l\left(l_{c}^{-1}\left(\frac{\partial V}{\partial W}, s\right), s\right)-\frac{\partial V}{\partial W} l_{c}^{-1}\left(\frac{\partial V}{\partial W}, s\right) \\
-\frac{1}{2} \frac{(\partial V / \partial W)^{2}\left(g(s)-r^{\prime}(s)\right)^{2}}{\left(\partial^{2} V / \partial W^{2}\right)\left[(1+\beta)^{2} \sigma^{2}(s)+\sigma_{e}^{2}(s)+2 \rho(1+\beta) \sigma(s) \sigma_{e}(s)\right]}=0, \\
V(W, T)=h(W, T) .
\end{gathered}
$$

It implies that the investor should borrow money from home bank and invest in foreign real project to get more profit in this case.

Case $2\left(\pi_{2}^{*}(s)<W(s) \leq \pi_{1}^{*}(s)\right)$. From Figure 2 , we can conclude that

$$
\pi^{*}(s)=W(s)
$$




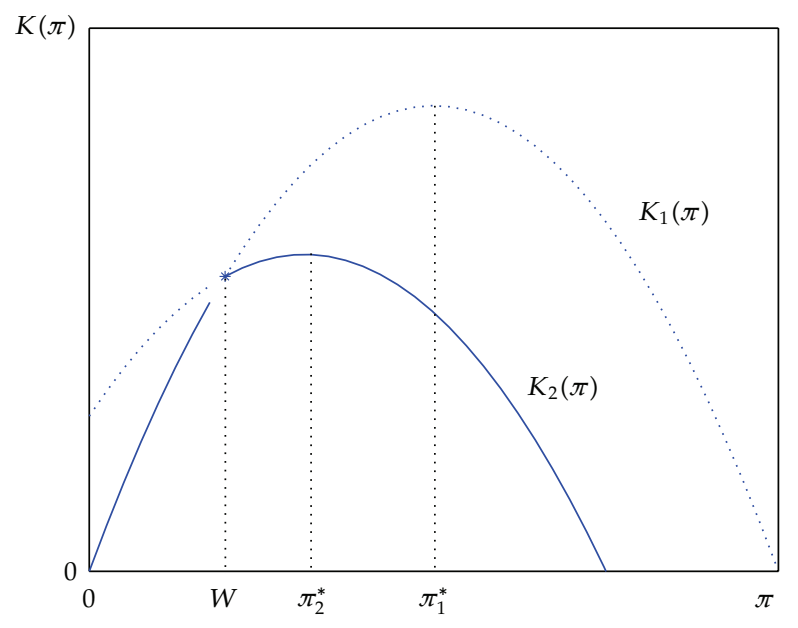

Figure 1: Case 1.

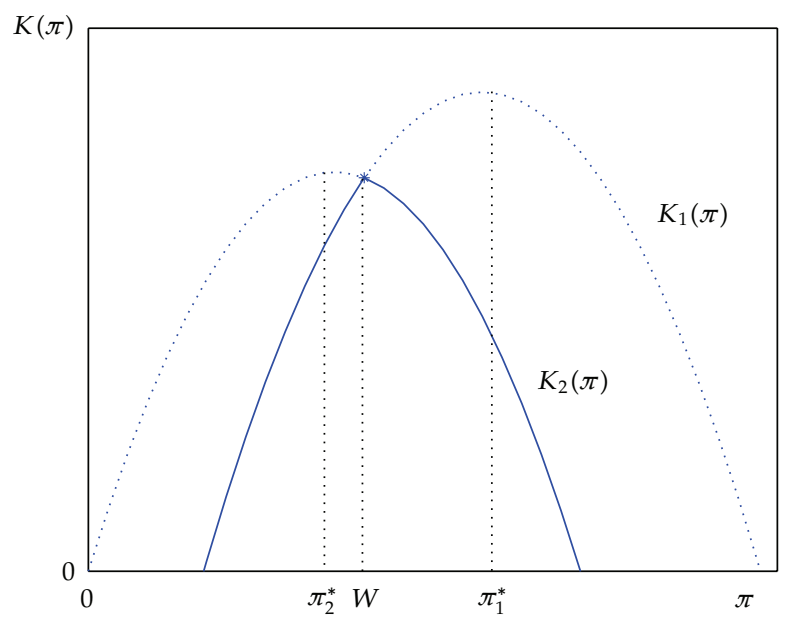

Figure 2: Case 2.

The corresponding HJB equation is

$$
\begin{gathered}
\frac{\partial V}{\partial s}+\frac{\partial V}{\partial W} g(s) W+l\left(l_{c}^{-1}\left(\frac{\partial V}{\partial W}, s\right), s\right)-\frac{\partial V}{\partial W} l_{c}^{-1}\left(\frac{\partial V}{\partial W}, s\right) \\
+\frac{1}{2} \frac{\partial^{2} V}{\partial W^{2}}\left[(1+\beta)^{2} \sigma^{2}(s)+\sigma_{e}^{2}(s)+2 \rho(1+\beta) \sigma(s) \sigma_{e}(s)\right] W^{2}=0, \\
V(W, T)=h(W, T) .
\end{gathered}
$$

It implies that the investor should put all his money into the foreign real project but without any loan in this situation. 


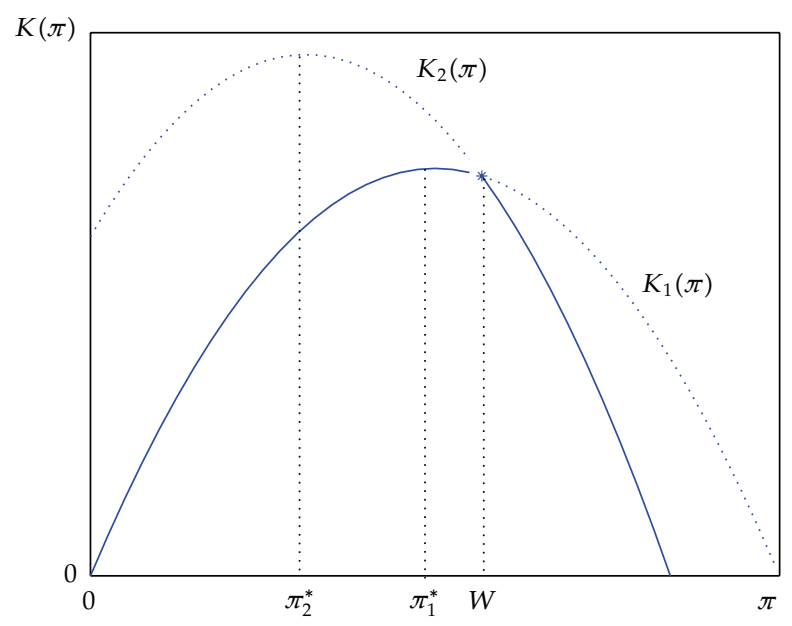

Figure 3: Case 3.

Case 3 (when $\pi_{1}^{*}(s)<W(s)$ ). From Figure 3, we can conclude that

$$
\pi^{*}(s)=\pi_{1}^{*}(s)
$$

The corresponding HJB equation is

$$
\begin{gathered}
\frac{\partial V}{\partial s}+\frac{\partial V}{\partial W} r(s) W+l\left(l_{c}^{-1}\left(\frac{\partial V}{\partial W}, s\right), s\right)-\frac{\partial V}{\partial W} l_{c}^{-1}\left(\frac{\partial V}{\partial W}, s\right) \\
-\frac{1}{2} \frac{(\partial V / \partial W)^{2}(g(s)-r(s))^{2}}{\left(\partial^{2} V / \partial W^{2}\right)\left[(1+\beta)^{2} \sigma^{2}(s)+\sigma_{e}^{2}(s)+2 \rho(1+\beta) \sigma(s) \sigma_{e}(s)\right]}=0, \\
V(W, T)=h(W, T) .
\end{gathered}
$$

It means that the investor should invest some wealth in foreign real project while puting some of his money in home bank account to reduce the risk in this case.

Now we proceed to give the economic analysis for the optimal investment portfolio.

Denote that

$\lambda=\frac{\pi^{*}(s)}{W(s)}, \quad A=-\frac{\left(\partial^{2} V / \partial W^{2}\right) W}{\partial V / \partial W}, \quad S_{\omega}^{2}(s)=(1+\beta)^{2} \sigma^{2}(s)+\sigma_{e}^{2}(s)+2 \rho(1+\beta) \sigma(s) \sigma_{e}(s)$.

Then $\lambda$ is the optimal proportion of the total wealth invested in the real project. $A$ corresponds to the Pratt-Arrow measure of relative risk aversion which indicates the investor's attitude to the risk in the investment and $S_{\omega}^{2}(s)$ refers to the total volatility of the portfolio in the foreign project, which is affected by not only the variability of the price but also the volatility of the exchange rate and their correlation coefficient. 
We can rewrite the above result in the following form:

$$
\text { Case 1: } \begin{aligned}
\lambda_{1} & =-\frac{\partial V / \partial W}{\left(\partial^{2} V / \partial W^{2}\right) W} \cdot \frac{g(s)-r^{\prime}(s)}{(1+\beta)^{2} \sigma^{2}(s)+\sigma_{e}^{2}(s)+2 \rho(1+\beta) \sigma(s) \sigma_{e}(s)} \\
& =\frac{1}{A S_{\omega}^{2}(s)}\left(g(s)-r^{\prime}(s)\right),
\end{aligned}
$$

Case 2: $\quad \lambda_{2}=1$,

Case 3: $\quad \lambda_{3}=\frac{1}{A S_{\omega}^{2}(s)}(g(s)-r(s))$.

For the Cases 1 and 3, the optimal proportion $\lambda$ can be referred as the speculative demand, which depends on the measure of relative risk aversion. Obviously, the optimal proportion is influenced by the variability of the portfolio. When $S_{\omega}^{2}(s)$ increases, $\lambda$ will decrease, that is, higher volatility in the foreign corporate market will simulate the investor to put more money in home riskless investment, that is, greater variability in oversea market can avoid home capital to flow out. On the other hand, the optimal proportion in the foreign corporate project is affected by whether the expectation rate of the cash flow in foreign project is higher than the interest (borrowing) rate in the bank.

\section{Special Case and Simulation}

In this section, we will take a typical utility function (CRRA case) to get the explicit solution to the optimal portfolio choice and give the simulation to show the influence of the variability parameters in the market.

The CRRA utility function is

$$
l(c, t)=L e^{-\gamma t} \frac{c^{1-R}}{1-R}, \quad h(W, t)=K \frac{W^{1-R}}{1-R}
$$

where $L$ and $K$ are constants, $R \in(0,1)$, and $\gamma>0$ is the discount factor. It is easily calculated that

$$
\begin{gathered}
c^{*}(s)=\left(\frac{1}{L} e^{\gamma s} \frac{\partial V}{\partial W}\right)^{-1 / R}, \\
\sup _{C}\left(l(c(s), s)-\frac{\partial V}{\partial W} c(s)\right)=\frac{R}{1-R}\left(L e^{-\gamma s}\right)^{1 / R}\left(\frac{\partial V}{\partial W}\right)^{(R-1) / R} .
\end{gathered}
$$

Since the utility function has regular form, here we use a conjecture method arising form the classical LQ problems to solve this problem. We first conjecture that the value function is of a special form, then solve the HJB equation via ordinary differential equations. 
In Case 1 , let us conjecture that $V(W, s)=\Phi_{1}(s)\left(W^{1-R} /(1-R)\right)$. The HJB equation can be rewritten as an Bernoulli type,

$$
\begin{gathered}
\Phi_{1}^{\prime}(s)+(1-R)\left[r^{\prime}(s)+\frac{\left(g(s)-r^{\prime}(s)\right)^{2}}{2 R\left[(1+\beta)^{2} \sigma^{2}(s)+\sigma_{e}^{2}(s)+2 \rho(1+\beta) \sigma(s) \sigma_{e}(s)\right]}\right] \Phi_{1}(s) \\
+R\left(L e^{-\gamma s}\right)^{1 / R}\left[\Phi_{1}(s)\right]^{(R-1) / R}=0, \\
\Phi_{1}(T)=K .
\end{gathered}
$$

Solving this equation, we can get

$$
\Phi_{1}(s)=e^{\int_{s}^{T} m_{1}(t) d t}\left[K^{1 / R}+\frac{1}{R} \int_{s}^{T} m_{2}(t) e^{-(1 / R) \int_{t}^{T} m_{1}(r) d r} d t\right]^{R}
$$

Here

$$
\begin{aligned}
& m_{1}(s)=(1-R)\left[r^{\prime}(s)+\frac{\left(g(s)-r^{\prime}(s)\right)^{2}}{2 R\left[(1+\beta)^{2} \sigma^{2}(s)+\sigma_{e}^{2}(s)+2 \rho(1+\beta) \sigma(s) \sigma_{e}(s)\right]}\right] \\
& m_{2}(s)=R\left(L e^{-\gamma s}\right)^{1 / R} .
\end{aligned}
$$

Then the explicit optimal solution to the first case is

$$
\begin{gathered}
V(W, s)=\Phi_{1}(s) \frac{W^{1-R}}{1-R}, \\
c^{*}(s)=\left[\frac{1}{L} e^{r s} \Phi_{1}(s)\right]^{-1 / R} W(s), \\
\pi^{*}(s)=\frac{g(s)-r^{\prime}(s)}{R\left[(1+\beta)^{2} \sigma^{2}(s)+\sigma_{e}^{2}(s)+2 \rho(1+\beta) \sigma(s) \sigma_{e}(s)\right]} W(s) .
\end{gathered}
$$

Since $W(s) \leq \pi_{2}^{*}(s)$, we have $\left(g(s)-r^{\prime}(s)\right) / R\left[(1+\beta)^{2} \sigma^{2}(s)+\sigma_{e}^{2}(s)+2 \rho(1+\beta) \sigma(s) \sigma_{e}(s)\right]$ $\geq 1$, that is, $r^{\prime}(s) \leq g(s)-R\left[(1+\beta)^{2} \sigma^{2}(s)+\sigma_{e}^{2}(s)+2 \rho(1+\beta) \sigma(s) \sigma_{e}(s)\right]$.

Similarly, solving (3.12) and (3.14) for Cases 2 and 3, respectively, we have

$$
\Phi_{2}(s)=e^{\int_{s}^{T} n_{1}(r) d r}\left[K^{1 / R}+\frac{1}{R} \int_{s}^{T} n_{2}(t) e^{-(1 / R) \int_{t}^{T} n_{1}(r) d r} d t\right]^{R},
$$


with

$$
\begin{gathered}
n_{1}(s)=(1-R)\left\{g(s)-\frac{1}{2} R\left[(1+\beta)^{2} \sigma^{2}(s)+\sigma_{e}^{2}(s)+2 \rho(1+\beta) \sigma(s) \sigma_{e}(s)\right]\right\} \\
n_{2}(s)=R\left(L e^{-\gamma s}\right)^{1 / R}, \\
\Phi_{3}(s)=e^{\int_{s}^{T} p_{1}(r) d r}\left[K^{1 / R}+\frac{1}{R} \int_{s}^{T} p_{2}(t) e^{-(1 / R) \int_{t}^{T} p_{1}(r) d r} d t\right]^{R},
\end{gathered}
$$

with

$$
\begin{aligned}
& p_{1}(s)=(1-R)\left[r(s)+\frac{\left(g(s)-r^{\prime}(s)\right)^{2}}{2 R\left[(1+\beta)^{2} \sigma^{2}(s)+\sigma_{e}^{2}(s)+2 \rho(1+\beta) \sigma(s) \sigma_{e}(s)\right]}\right] \\
& p_{2}(s)=R\left(L e^{-\gamma s}\right)^{1 / R} .
\end{aligned}
$$

Finally we draw the following conclusion.

Proposition 4.1. Under all the above assumptions, the optimal strategies to the optimal portfolio and consumption choice problem (2.11)-(2.12) for the specific CRRA case is given by

$$
\begin{aligned}
& \pi^{*}(s)= \begin{cases}\frac{g(s)-r^{\prime}(s)}{R S_{\omega}^{2}(s)} W(s), & r^{\prime}(s) \leq g(s)-R S_{\omega}^{2}(s), \\
W(s), & r(s)<g(s)-R S_{\omega}^{2}(s)<r^{\prime}(s), \\
\frac{g(s)-r(s)}{R S_{\omega}^{2}(s)} W(s), & r(s) \geq g(s)-R S_{\omega}^{2}(s),\end{cases} \\
& c^{*}(s)= \begin{cases}\left(\frac{1}{L} e^{r s} \Phi_{1}(s)\right)^{-1 / R} W(s), & r^{\prime}(s) \leq g(s)-R S_{\omega}^{2}(s), \\
\left(\frac{1}{L} e^{r s} \Phi_{2}(s)\right)^{-1 / R} W(s), & r(s)<g(s)-R S_{\omega}^{2}(s)<r^{\prime}(s), \\
\left(\frac{1}{L} e^{r s} \Phi_{3}(s)\right)^{-1 / R} W(s), & r(s) \geq g(s)-R S_{\omega}^{2}(s) .\end{cases}
\end{aligned}
$$


Furthermore, the value function is given by

$$
V(W, s)= \begin{cases}\Phi_{1}(s) \frac{W^{1-R}}{1-R}, & r^{\prime}(s) \leq g(s)-R S_{\omega}^{2}(s), \\ \Phi_{2}(s) \frac{W^{1-R}}{1-R}, & r(s)<g(s)-R S_{\omega}^{2}(s)<r^{\prime}(s), \\ \Phi_{3}(s) \frac{W^{1-R}}{1-R}, & r(s) \geq g(s)-R S_{\omega}^{2}(s),\end{cases}
$$

where $\Phi_{1}(s), \Phi_{2}(s)$, and $\Phi_{3}(s)$ satisfy (4.4), (4.7), and (4.9), respectively.

We can also give one conclusion which has explicit economics interpretation.

Proposition 4.2. In CRRA case, we can get from simple calculus that the Pratt-Arrow measure of relative risk aversion $A=R, R \in(1,+\infty)$.

Remark 4.3. The constant $R$ in CRRA utility function can indicate the investor's attitude to the risk in the market.

From the history of price data in the market, we can use statistical method to estimate the parameters in the model. The coefficients in the utility function can be determined by personal preference. Then we can use the above result to give an optimal investment choice for an investor in CRRA case. Now let us give a simulating example. In this example, let the coefficients be constants for simplicity, and we only consider the choice at initial time $t=0$.

Example 4.4. Suppose an investor wants to make a corporate investment, but domestic investment may face much more competitions or cannot be executed due to certain policy barriers or monopoly restriction, such as crude oil exploitation in China. However, international corporate investment (e.g., to build a factory in some other countries) may be possible and profitable in some emerging markets due to the advantages in techniques, in human resources, and so on. The factory will produce and sell some products whose price is evaluated in foreign currency, for example, in US dollar. Meanwhile, he also needs to seek a domestic bond to catch a fundamental yield and avoid risk.

Here we just take the following parameters depending on the situation of the real market. Set $S(0)=4, P(0)=3, \beta=-0.4, \alpha_{s}=0.13, \alpha_{p}=0.1, R=0.5, L=0.8, T=2, K=0.9$, $r=0.04, r^{\prime}=0.1, \rho=0.1$, and $\alpha_{e}=0.2$.

From formula (4.11), we can draw the conclusion that the optimal proportion decreases when the volatility parameter $\sigma$ or $\sigma_{e}$ increases. And also the optimal proportion should be divided into three parts according to the different volatility. Let us first fix one of $\sigma$ and $\sigma_{e}$ and give the relationship between $\pi^{*} / W$ and another one.

In Figures 4 and 5, the curve goes just as we have expected.

Fixed $\sigma_{e}=0.3$ (see Figure 4), when $\sigma \leq 0.893, \pi^{*} / W$, decreases down to 1 . That means that high volatility in foreign corporate project market can stimulate to the riskless investment in home bond.

However, when the volatility is not too big and the expected reward of the foreign project is higher than the bond, the investor can borrow money from home bank and invest all his wealth into the project. Taking $\sigma=0.663$, for example, here $\pi^{*} / W=1.67$. That is, 


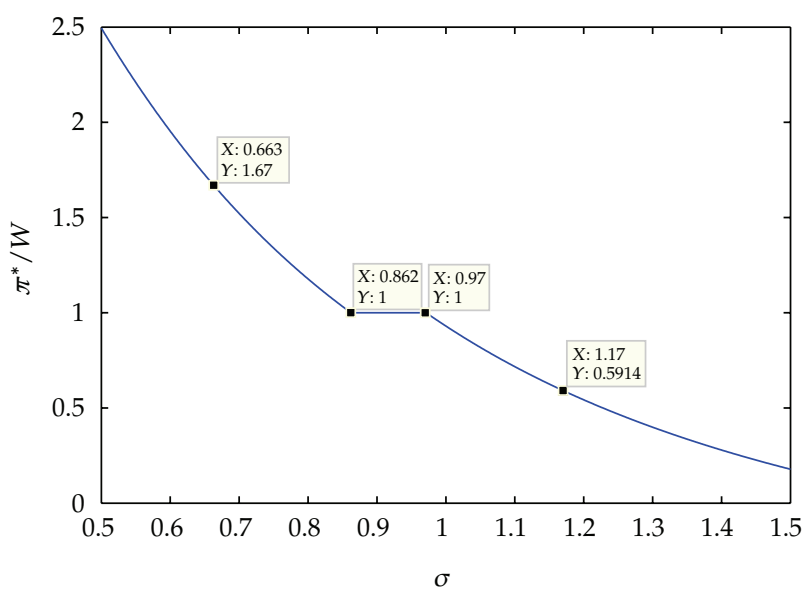

Figure 4: Relationship between $\sigma$ and $\pi^{*} / W$.

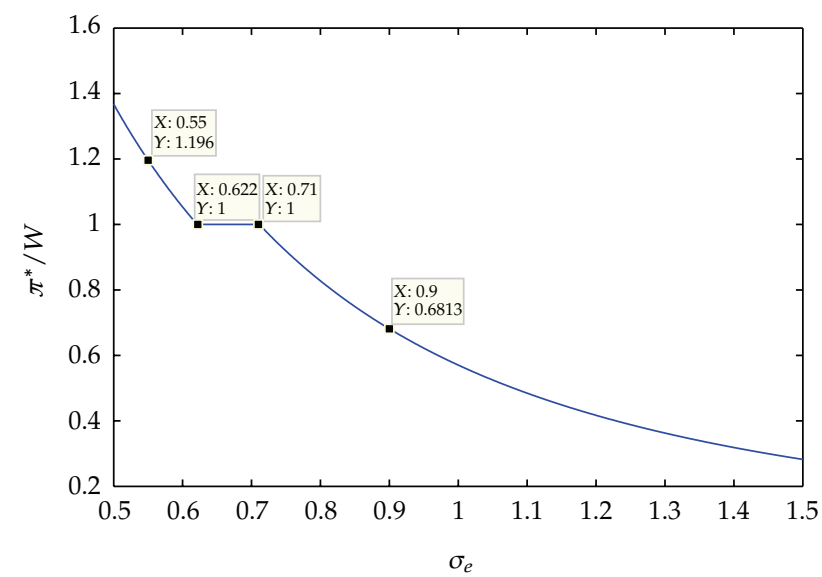

Figure 5: Relationship between $\sigma_{e}$ and $\pi^{*} / W$.

she or he can borrow from home bank an amount about two thirds of his wealth to invest in the foreign project. When $0.862 \leq \sigma \leq 0.97, \pi^{*} / W=1$. This implies that the investor should better put all his money to the foreign project but without any loan. When $\sigma \geq 0.97, \pi^{*} / W$ continue decreasing. Taking $\sigma=1.17$, then $\pi^{*} / W=0.5914$. This shows the investor should better save about two fifths of his money in the home bank and invest the money left in the foreign project.

For fixed $\sigma=0.5$ (see Figure 5), we can get a similar result. The high volatility in foreign exchange rate market can avoid home capital to flow out. In this case, when $0.622 \leq$ $\sigma_{e} \leq 0.71, \pi^{*} / W=1$. We also take several points for example. When $\sigma_{e}=0.55, \pi^{*} / W=1.196$, that is, the investor's optimal choice is to borrow about twenty percents of his wealth and put all money into the foreign project.

The volatility in exchange rate between different currencies gives people a chance to get higher reward. We can take advantage of the difference created by the volatility just like that of a stock. But risk also comes along with the volatility. When one currency has a high 


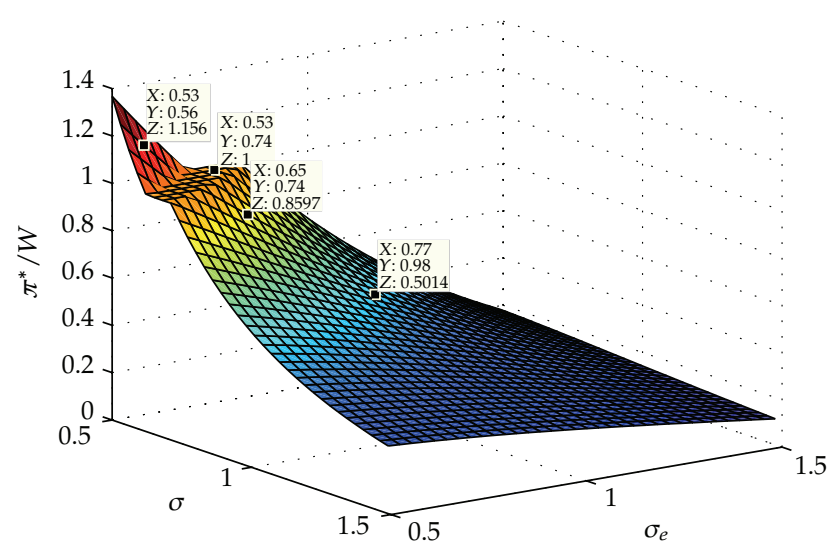

Figure 6: Relationship between $\sigma, \sigma_{e}$, and $\pi^{*} / W$.

volatility, it indicates that this kind of currency is not stable or even the economy of a country is not stable, so it is better not do any investment in that country. Figure 6.

We also give the variance tendency of $\pi^{*} / W$ with respect to both $\sigma$ and $\sigma_{e}$. See

It tells us that the optimal proportion is a nonincreasing function of both $\sigma$ and $\sigma_{e}$. When $\sigma=0.53, \sigma_{e}=0.56$, then $\pi^{*} / W=1.156$, and at this time the investor can borrow money to invest in the foreign project. When $\sigma=0.53, \sigma_{e}=0.74$, and the volatility of the foreign exchange rate is higher, we have $\pi^{*} / W=1$. When $\sigma=0.77, \sigma_{e}=0.98, \pi^{*} / W=0.5014$. At this time, the investor has to put about half of his wealth in home bank. This coincides with the above economic analysis for the volatility of exchange rate.

\section{Conclusions}

In this paper, we have considered one kind of investment and consumption choice problems for an investor who can invest her or his wealth either in a domestic bond or in an oversea real project. From Proposition 3.1, we knew that the classical Dynamical Programming Principle still holds for this optimal problem. The optimal investment and consumption choice have also been achieved by solving the corresponding H-J-B equation and the economic analysis was then given for that. For a typical CRRA utility function case, we got the explicit optimal investment and consumption strategy and then some simulation results illustrating the influence of market's volatility parameters to the optimal choice. We think similar idea may also be applied to consider the optimal auditing problem in bank management, since for the manager of a bank, he/she should decide the optimal capital allocation not only to make the maximal profit, but also to meet the auditing request according to Basel II. See Bosch et al. [17] and Petersen et al. [18] for more details.

One of the future tasks is to extend our model to a more general case, for instance, solving the problem under convex restriction which coincides better with the situation of the real market, generalizing the utility function to a more general case and so on. In addition, there are several interesting problems about the underlying topic, such as the corresponding convex restriction problem and the partial information problem. Since these problems are more consistent with the practical situation in real market, we will furthermore investigate 
them in further work and then desire to solve some practical optimization investment problems in financial market.

\section{Acknowledgments}

The authors would like to thank the editor and anonymous referee for many helpful comments and suggestions which have led to the improved version. This paper is supported by NSFC (10921101, 10926098), National Basic Research Program of China (973 Program) (2007CB814904), Shandong Provincial Natural Science Foundation (JQ200801 and 2008BS01024), the Postdoctoral Innovation Fund of Shandong Province (200903078), and IIFSDU (2009).

\section{References}

[1] J. J. Choi, "Diversification, exchange risk, and corporate international investment," Journal of International Business Studies, pp. 145-155, 1989.

[2] D. Duffie, Dynamic Asset Picing Theory, Princeton University Press, Princeton, NJ, USA, 1992.

[3] R. Merton, Continuous-Time Finance, Blackwell, Oxford, Uk, 1991.

[4] T. Zariphopoulou, "Investment-consumption models with transaction fees and Markov-chain parameters," SIAM Journal on Control and Optimization, vol. 30, no. 3, pp. 613-636, 1992.

[5] T. Zariphopoulou, "Consumption-investment models with constraints," SIAM Journal on Control and Optimization, vol. 32, no. 1, pp. 59-85, 1994.

[6] M. Bellalah and Z. Wu, "A simple model of corporate international investment under incomplete information and taxes," Annals of Operations Research, vol. 165, no. 1, pp. 123-143, 2009.

[7] G. C. Wang and Z. Wu, "General maximum principles for partially observed risk-sensitive optimal control problems and applications to finance," Journal of Optimization Theory and Applications, vol. 141, no. 3, pp. 677-700, 2009.

[8] N. El Karoui, S. Peng, and M. C. Quenez, "Backward stochastic differential equations in finance," Mathematical Finance, vol. 7, no. 1, pp. 1-71, 1997.

[9] R. Bellman, "On the theory of dynamic programming," Proceedings of the National Academy of Sciences of the United States of America, vol. 38, pp. 716-719, 1952.

[10] J. Mukuddem-Petersen and M. A. Petersen, "Bank management via stochastic optimal control," Automatica, vol. 42, no. 8, pp. 1395-1406, 2006.

[11] J. Mukuddem-Petersen and M. A. Petersen, “Optimizing asset and capital adequacy management in banking," Journal of Optimization Theory and Applications, vol. 137, no. 1, pp. 205-230, 2008.

[12] I. Karatzas, "Optimization problems in the theory of continuous trading," SIAM Journal on Control and Optimization, vol. 27, no. 6, pp. 1221-1259, 1987.

[13] R. Bellman, Dynamic Programming, Princeton University Press, Princeton, NJ, USA, 1957.

[14] S. G. Peng, "A generalized dynamic programming principle and Hamilton-Jacobi-Bellman equation," Stochastics and Stochastics Reports, vol. 38, no. 2, pp. 119-134, 1992.

[15] N. El Karoui and M.-C. Quenez, "Dynamic programming and pricing of contingent claims in an incomplete market," SIAM Journal on Control and Optimization, vol. 33, no. 1, pp. 29-66, 1995.

[16] J. Yong and X. Y. Zhou, Stochastic Control: Hamiltonian Systems and HJB Equations, vol. 43 of Applications of Mathematics, Springer, New York, NY, USA, 1999.

[17] T. Bosch, J. Mukuddem-Petersen, M. A. Petersen, and I. Schoeman, “Optimal auditing in the banking industry," Optimal Control Applications E Methods, vol. 29, no. 2, pp. 127-158, 2008.

[18] M. A. Petersen, M. C. Senosi, and J. Mukuddem-Petersen, Subprime Mortgage Models, Nova, New York, NY, USA, 2010. 


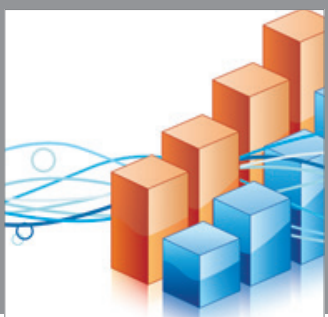

Advances in

Operations Research

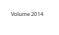

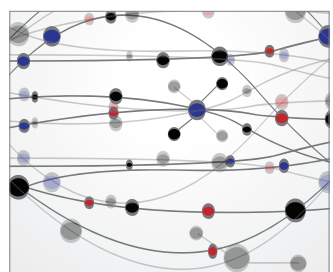

\section{The Scientific} World Journal
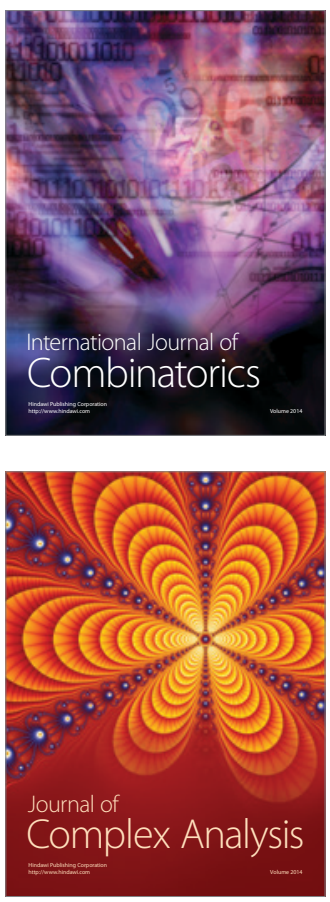

International Journal of

Mathematics and

Mathematical

Sciences
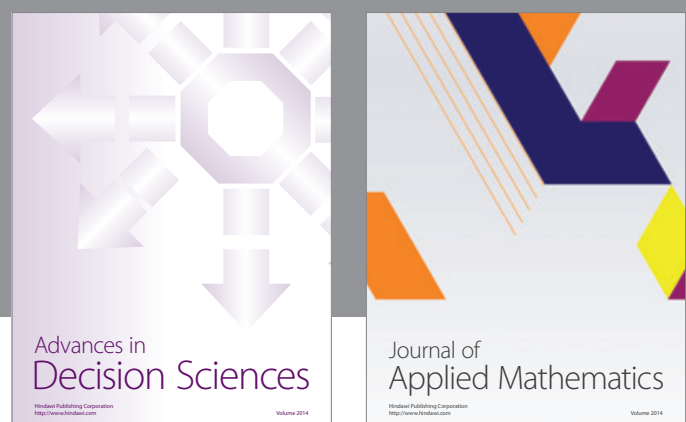

Journal of

Applied Mathematics
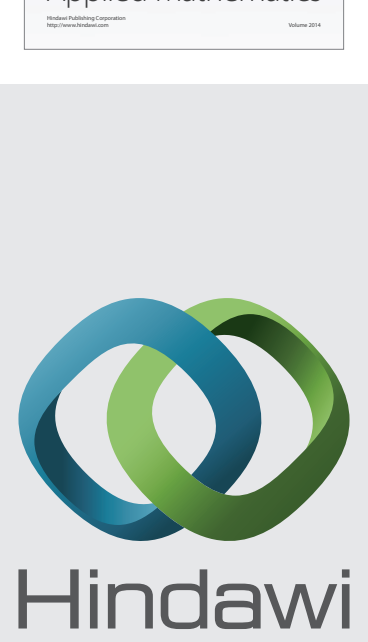

Submit your manuscripts at http://www.hindawi.com
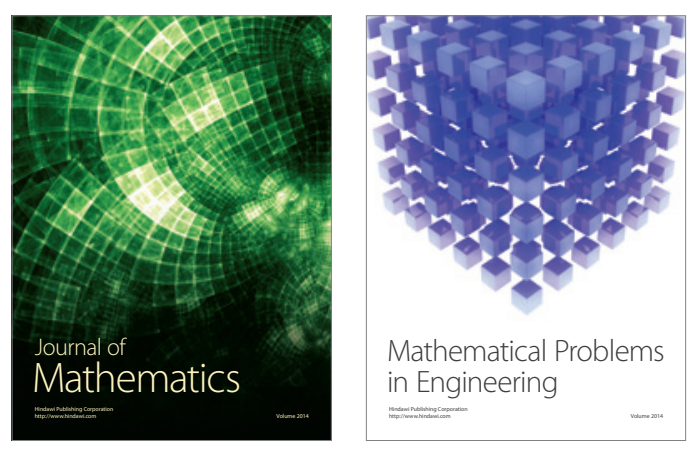

Mathematical Problems in Engineering
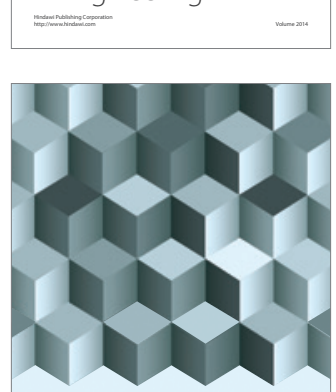

Journal of

Function Spaces
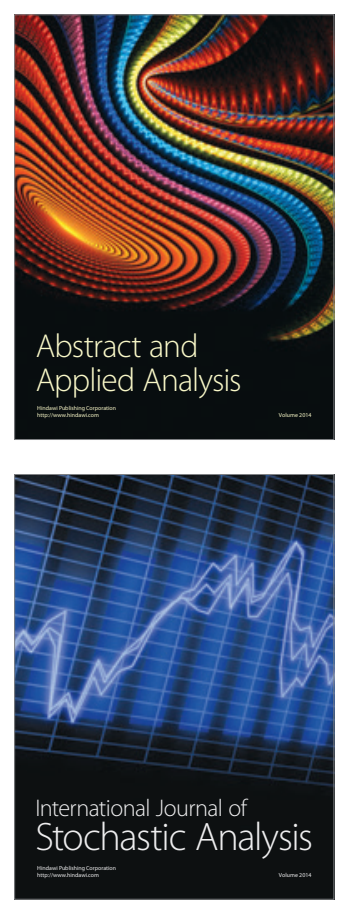

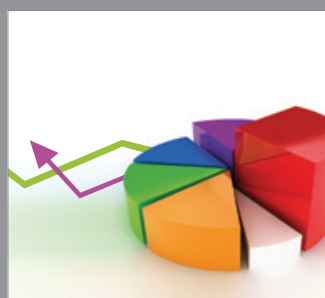

ournal of

Probability and Statistics

Promensencen
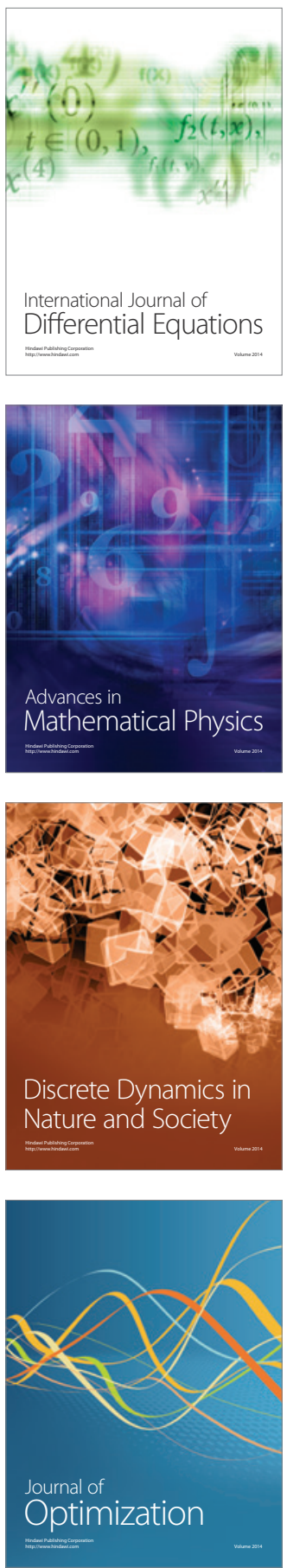